\title{
Comparison between SuperDARN flow vectors and equivalent ionospheric currents from ground magnetometer arrays
}

\author{
J. M. Weygand, ${ }^{1}$ O. Amm, ${ }^{2}$ V. Angelopoulos, ${ }^{3}$ S. E. Milan, ${ }^{4}$ A. Grocott, ${ }^{4}$ H. Gleisner, ${ }^{5}$ \\ and C. Stolle ${ }^{6}$ \\ Received 23 November 2011; revised 9 April 2012; accepted 10 April 2012; published 25 May 2012.
}

[1] Equivalent ionospheric currents obtained with the spherical elementary current systems (SECS) method and derived from nearly 100 ground magnetometers spread over North America and Greenland are compared with ionospheric flow vectors measured by the SuperDARN radars during both the summer and winter seasons. This comparison is done over a range of spatial separations, magnetic latitudes, magnetic local times, and auroral electrojet activity to investigate under what conditions the vectors are anti-parallel to one another. Our results show that in general the equivalent ionospheric currents are anti-parallel to the flows and the best results are achieved within the auroral oval during active geomagnetic conditions in the dawn, dusk and noon sectors in the northern hemisphere summer. These results indicate the best anti-parallel alignment occurs when the currents and flows are large and well defined. Factors that may influence the alignment include ionospheric conductivity gradients and quiet time backgrounds. Our results can be used to approximate the macroscopic $(\sim 1000 \mathrm{~km})$ ionospheric convection patterns. The SECS maps represent a value-added product from the raw magnetometer database and can be used for contextual interpretation; they can help with our understanding of magnetosphere-ionosphere coupling mechanisms using ground arrays and the magnetospheric spacecraft data, and they can be used as input for other techniques.

Citation: J. M. Weygand, O. Amm, V. Angelopoulos, S. E. Milan, A. Grocott, H. Gleisner, and C. Stolle (2012), Comparison between SuperDARN flow vectors and equivalent ionospheric currents from ground magnetometer arrays, J. Geophys. Res., 117, A05325, doi:10.1029/2011JA017407.

\section{Introduction}

[2] Ground-based magnetometer observations are critical for deriving ionospheric current patterns and magnetosphereionosphere coupling studies. For decades magnetometers have been used to derive equivalent ionospheric currents [Chapman and Bartels, 1940; Untiedt et al., 1978; Amm and Viljanen, 1999], which are the divergence free ionospheric

\footnotetext{
${ }^{1}$ Institute of Geophysics and Planetary Physics, UCLA, Los Angeles, California, USA.

${ }^{2}$ Arctic Research Unit, Finnish Meteorological Institute, Helsinki, Finland.

${ }^{3}$ Department of Earth and Space Sciences, UCLA, Los Angeles, California, USA.

${ }^{4}$ Department of Physics and Astronomy, University of Leicester, Leicester, UK.

${ }^{5}$ Atmosphere Space Research Division, Danish Meteorological Institute, Copenhagen, Denmark.

${ }^{6}$ Technical University of Denmark, DTU Space, Copenhagen, Denmark.

Corresponding author: J. M. Weygand, Institute of Geophysics and Planetary Physics, University of California, 3845 Slichter Hall, Charles E. Young Drive, PO Box 951567, Los Angeles, CA 90095-1567, USA. (jweygand@igpp.ucla.edu)

Copyright 2012 by the American Geophysical Union. 0148-0227/12/2011JA017407
}

currents calculated from magnetic perturbations associated with the Pedersen and Hall currents. According to Fukushima's theorem [Fukushima, 1969, 1976] the magnetic effects on the ground due to the field aligned currents (FACs) are canceled out by the magnetic perturbations of the Pedersen currents for a uniformly conducting ionosphere and high inclination angles $\left(\sim 90^{\circ}\right)$. However, radar measurements and auroral images indicated that the ionosphere is not uniformly conducting [Kosch et al., 2000; Lummerzheim et al., 1991] and the Pedersen and FACs will play some role in the magnetic perturbation measured on the ground. The bulk of these magnetic fluctuations observed on the ground are mainly due to the ionospheric Hall currents. The Hall currents within the E region of the ionosphere according to Fukushima's theorem for a uniformly conducting, thin ionosphere are antiparallel to the ionospheric flow within the $\mathrm{F}$ region at about $120 \mathrm{~km}$ above the surface of the Earth [Fukushima, 1969, 1976; Lester et al., 1993]. Thus, a macroscopic picture of the ionospheric convection can be determined by assuming that the equivalent ionospheric currents are composed mainly of Hall currents [Amm, 1997] and assuming the ionosphere is thin and uniformly conducting, but this assumption should be investigated in more detail. 
[3] Using equivalent ionospheric currents to determine the ionospheric convection patterns is important because radar measurements of the ionospheric convection are not always available due to limited backscatter of the signal and cadence. Ground magnetometer measurements are not limited to ionospheric backscatter and provide a higher cadence. The use of magnetometer data to derive ionospheric convection patterns is not new [Kamide, 1988; Richmond et al., 1988; Lyatsky et al., 1999; Benkevitch et al., 2006], however, few studies have done a detailed comparison between the equivalent ionospheric currents and the closely associated magnetic equivalent convection, which is the ground equivalent currents determined by rotating the horizontal magnetic perturbation vector by $90^{\circ}$, with radar measurements of the ionospheric flow [Benkevitch et al., 2006]. No studies have compared the SuperDARN ionospheric convective flow with the equivalent ionospheric currents obtained from the spherical elementary current systems (SECS) method [Amm and Viljanen, 1999].

[4] Benkevitch et al. [2006] compared 5 days from 2001 of ionospheric convection data observed by the SuperDARN radars with the magnetic equivalent convection derived from ground magnetometer data recorded over most of the northern hemisphere. They limited their data set to radar measurements over $100 \mathrm{~m} / \mathrm{s}$ and magnetic perturbations greater than $5 \mathrm{nT}$ and removed a quiet time background of daily variations obtained by averaging the 5 quietest days from the magnetometer measurements. Their study demonstrated that the best agreement between the magnetic equivalent convection and the ionospheric convection occurred for the sunlit, summer ionosphere when the ionospheric conductivity is the highest and the worst agreement was during the dark, winter hemisphere when the conductivity is the lowest. Overall, the magnetic equivalent convection vector was within $45^{\circ}$ of the ionospheric convection vector in about $55 \%$ of the measurements and the worst agreement occurred in the dawn sector while the best agreement was in the dusk sector.

[5] In magnetic equivalent convection the magnetic field perturbations are rotated $90^{\circ}$ to obtain the approximate ionospheric convection direction while the equivalent ionospheric currents should be approximately anti-parallel or $180^{\circ}$ with respect to the ionospheric convection. In this study we will be using the SECS method to derive the equivalent ionospheric currents. The SECS technique defines two elementary current systems: a divergence-free elementary system with currents that flow entirely within the ionosphere and a curl-free system whose divergences represent the currents normal to the ionosphere. The superposition of these two elementary current systems with different weights (scaling factors) can reproduce any vector field on a sphere. If it is known a priori that the vector field is curl-free or divergencefree, then only one set of basis functions is needed, and thus $50 \%$ of the free coefficients (those associated with the other current system) can be eliminated. For more details on the SECS method, see Amm and Viljanen [1999]. Details on the application of this technique to the magnetometers located in North America and Greenland can be found in Weygand et al. [2011].

[6] The objective of this study is to compare the equivalent ionospheric currents derived from the SECS method with the ionospheric flow measured by SuperDARN and the motivation is to demonstrate the equivalent currents can be used to approximate the large scale ionospheric convection pattern. Furthermore, this study shows that the SECs method provides a better estimate of ionospheric convection pattern (via ionospheric equivalent currents) then simply rotating the magnetic field vectors by $90^{\circ}$ as was done by Benkevitch et al. [2006]. In the next section we will discuss the data and methodology. In the third section we will present our results using three months of magnetometer and radar data and in the last two sections we will discuss the importance of our results and summarize.

\section{Instrumentation and Data}

[7] The data for this study come from two distinct sources. The ionospheric flow vectors are derived from the Super Dual Auroral Radar Network (SuperDARN) and the equivalent ionospheric currents are derived from seven ground magnetometer arrays in North America and Greenland. During the intervals used in this study, data from 11 radars were available in the northern hemisphere and most of these radars take measurements over North America and Greenland. The radars operate in the high frequency bands between 8 and $22 \mathrm{MHz}$ and measure the Doppler velocity of plasma density irregularities in the ionosphere. In their standard operating mode each radar scans through 16 beams of azimuthal separation $3.24^{\circ}$, with a total scan time of $1 \mathrm{~min}$. Each of the beams is divided into 75 range gates of length $45 \mathrm{~km}$, and so in each full scan the radars each cover $52^{\circ}$ in azimuth and over $3000 \mathrm{~km}$ in range [Greenwald et al., 1995; Chisham et al., 2007]. For this study we are using the gridded velocity values and the spatial resolution of the flow vectors is on the order of $1^{\circ}$ in latitude and $2^{\circ}$ in longitude and the temporal resolution is $2 \mathrm{~min}$. The longitudinal resolution of the gridded data varies with latitude to maintain an approximately square grid cell. To obtain these values from the data for each radar some median filtering is done to get rid of noise and a simple radio wave propagation model is applied to calculate the latitude and longitude where each of the line of sight velocities originated. Each of the line of sight velocities of a radar is then placed into the grid-bin. If a grid-bin contains more than one measurement from the same radar, then the median of all measurements is saved. We then combine all grid files from all radars into one grid. The approximate uncertainty in the azimuth and magnitude is about 1\% [Ruohoniemi and Baker, 1998; L. Clausen, private communication, 2010]. Since the radars only measure lineof-sight velocities, all the measurements in a grid are used to constrain a global model of the full 2-D convection pattern. A set of line of sight velocities derived from the model are compared to the measurements and the sum of the squares of their differences is minimized using the method of singular value decomposition [e.g., Press et al., 1992]. The best fit vector velocities derived by this technique, which is described in detail by Ruohoniemi and Baker [1998], are what we use in the present study. As a result of the global nature of the fitting, errors may be introduced in the determination of the local flows even in regions of good radar data coverage. The errors associated with the fitting are smaller than the approximate uncertainty associated with the determination of the azimuth and magnitude, which we noted above is on the order of $1 \%$. 
THEMIS EICs: 09-Jan-2008 00:08:00
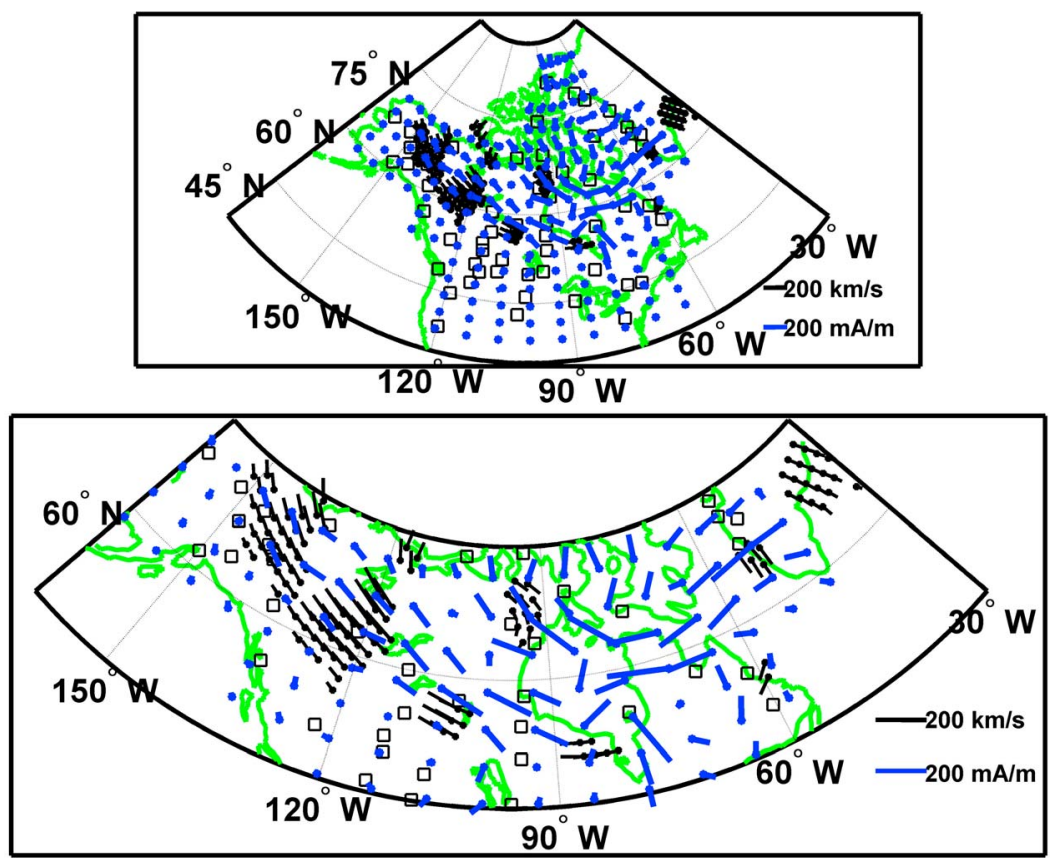

Figure 1. Example from January 9th, 2008 at 00:08:00 UT of typical anti-parallel alignment and misalignment between equivalent ionospheric currents and SuperDARN flows. The AE index is moderate during this period at about $150 \mathrm{nT}$. (top) The region over which we can calculate equivalent ionospheric currents, which includes North America and the west coast of Greenland. (bottom) A blown up portion of that area to better indicate the anti-parallel alignment at about $60^{\circ}$ geographic latitude and about $120^{\circ}$ west geographic longitude and the misalignment at about $65^{\circ}$ geographic latitude and about $55^{\circ}$ west geographic longitude. The current scale in the bottom panel is the same as the top panel.

[8] For this study we have obtained data from seven different ground magnetometer arrays: CANMOS (Canadian Magnetic Observatory System) (http://geomag.nrcan.gc.ca/ obs/canmos-eng.php), CARISMA (Canadian Array for Real time Investigations of Magnetic Activity) [Mann et al., 2008], GIMA (Geophysical Institute Magnetometer Array) (http:// www.asf.alaska.edu/program/gdc/project/magnetometer), Technical University of Denmark (DTU) Magnetometer Ground Stations in Greenland (http://www.space.dtu.dk/ MagneticGroundStations.aspx), MACCS (Magnetometer Array for Cusp and Cleft Studies) [Engebretson et al., 1995], the STEP (Solar-Terrestrial Energy Program) magnetometer array (http://step-p.dyndns.org/ khay/), and THEMIS GMAG (Time History of Events and Macroscale Interactions During Substorms Ground Magnetometers) [Russell et al., 2008]. Many of the ground magnetometer arrays share some stations. All of the data from GIMA, MACCS, and Greenland stations used in this study can be obtained from the THEMIS GMAG online data archive, while the rest were obtained from the original provider. In total we have the potential of obtaining data from nearly 100 different stations at this time. We have not included the Greenland stations on the East coast because these stations are located far from the rest of the ground magnetometers. Figure 1a displays the distribution of the stations used in this study.

[9] The SECS are calculated with the available ground magnetometer data. The number of available stations may change from day to day due to data gaps, changes in baseline, and measurement errors. The spatial resolution of the equivalent ionospheric currents is about $2.9^{\circ}$ in geographic latitude and $6.9^{\circ}$ degrees geographic longitude. The temporal resolution for this data set is $10 \mathrm{~s}$. More details on the calculation of the SECS and the description of the SECS over North America and Greenland can be found in Amm and Viljanen [1999] and Weygand et al. [2011].

\section{Results}

[10] Figure $1 \mathrm{~b}$ displays a good example of the typical antiparallel alignment and misalignments observed between the equivalent ionospheric vectors (blue vectors) and the SuperDARN flow vectors (black vectors) for near simultaneous measurements over North America on January 9th, 2008 at 00:08:00 UT in the dusk sector. By "nearly simultaneous" we refer to the $1 \mathrm{~min}$ period it takes the SuperDARN radars to acquire data over the full field of view. The equivalent ionospheric current data has no integration time. At about $60^{\circ}$ geographic latitude and about $120^{\circ}$ west geographic longitude and continuing over to the Alaska Canada boarder there is good anti-parallel alignment between the currents and the flows in areas that are close to ground magnetometer stations (black boxes) and areas farther from ground stations. However, at about $65^{\circ}$ geographic latitude and about $55^{\circ}$ west geographic longitude near the Maniitsoq, Greenland magnetometer the flows and ionospheric currents are nearly perpendicular to one another despite the fact that the measurements were made near the ground magnetometer station. 


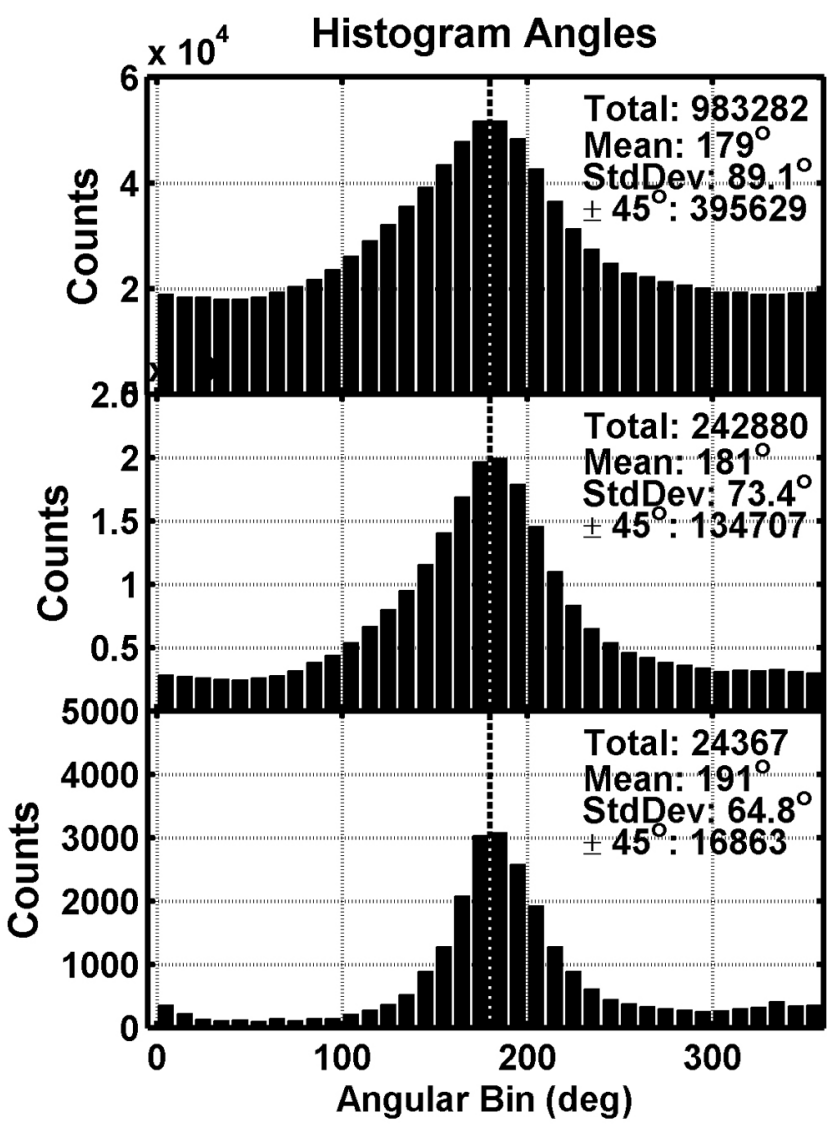

Figure 2. Histograms of angle between equivalent ionospheric currents and SuperDARN flow vectors. (top) The distribution for all near simultaneous vectors. (middle) Only flow magnitudes larger than $100 \mathrm{~m} / \mathrm{s}$ and currents larger than $46.2 \mathrm{~mA} / \mathrm{m}$. (bottom) Angles for currents and flows located close to ground magnetometer stations. In each distribution in the upper right corner is the total number of points within the distribution, the mean angles, the standard deviations, and the total number of points with $45^{\circ}$ of the anti-parallel direction.

This $90^{\circ}$ phase difference is not typical for that region in Greenland and is most likely not due to ground conductivity and the salt water. Why the measurements do not agree at this location is unclear. We speculate that the approximate $90^{\circ}$ difference in the alignment is due to ionospheric conductivity gradients, but we have no proof to support this hypothesis.

[11] With the nearly simultaneous magnetometer and ionosphere flow measurements we construct histograms of the angles between the equivalent ionospheric current vectors and the flow vectors. To determine the angle we take the arctangent of the current vector crossed with the nearest neighbor flow vector within about $1.5^{\circ}$ in latitude and about $3.5^{\circ}$ in longitude divided by the current vector dotted with the same flow vector. Figure 2 consists of histograms of the angle between the current vectors and the SuperDARN flow vectors using three months (June 2007, December 2007, and January 2008) of nearly simultaneous current and flow vectors. In Figure 2 (top) we show the distribution of all angles available, which totals to almost 1 million angles with no requirements on the current and flow magnitudes and the largest spatial separation between the current vector and the flow vector location is about $1.5^{\circ}$ in latitude and about $3.5^{\circ}$ in longitude. Limiting the spatial separation between the flow and current vectors further does not significantly improve the standard deviation and location of the peak of the distribution. Also, given in the upper right corner is the mean angle, which is $179^{\circ}$; the standard deviation, which is about $89^{\circ}$, to indicate the spread of the measurements; and the number of measurements within $45^{\circ}$ of anti-parallel alignment, which consists of about $1 / 3$ of the total number of angles. While the mean indicates that on average the vectors are anti-parallel, there is a very large noise threshold producing a peak to background ratio of about 2.5. Here we have defined the peak to background ratio to be the ratio of the counts at the peak of the distribution to an average noise threshold level determined from the counts in the bins between $0^{\circ}$ to $50^{\circ}$ and $310^{\circ}$ to $360^{\circ}$ ((maximum peak value) $/$ (mean background value)).

[12] In order to increase the peak to background ratio in Figure 2 (top) we constructed histograms (not shown) of the equivalent ionospheric current magnitudes and flow magnitudes to determine which current and flow values are small and therefore close to the limitations of the techniques. From the histogram of the currents we determined a mean of the distribution to be $46.2 \mathrm{~mA} / \mathrm{m}$ and all currents below this value are considered too small to include in our study. For the histogram of the flow magnitudes we found a mean value slightly less than $100 \mathrm{~m} / \mathrm{s}$ which is similar to the value of $100 \mathrm{~m} / \mathrm{s}$ used in the Benkevitch et al. [2006] study. To be consistent with the Benkevitch et al. [2006] study, we use a value of $100 \mathrm{~m} / \mathrm{s}$.

[13] Figure 2 (middle) is the histogram of the angles after removing the small equivalent ionospheric current and flow magnitudes. Removing these small values significantly reduces the number of available measurements to about 240,000 , but also significantly reduces the standard deviation to $73.4^{\circ}$ while increasing the peak to background ratio to about 10 and still maintaining an average anti-parallel alignment. Furthermore, nearly half the available measurements are now within $45^{\circ}$ of anti-parallel alignment.

[14] However, to reliably compare the results of the SECS technique with those of magnetic convection technique used in the Benkevitch et al. study we also will consider only those measurements obtained within about $1.5^{\circ}$ latitude and $3.5^{\circ}$ longitude of a ground magnetometer station. These ranges are based on half the distance between grid points for the equivalent ionospheric currents.

[15] Figure 2 (bottom) shows the distribution of the angles for only measurements close to the ground magnetometer stations. The purpose of this bottom panel is to focus on currents determined closest to the magnetometer station and presumably the most reliable. Furthermore, we want to reproduce the procedure similar to that used to create Figures 4-6 in the Benkevitch et al. [2006] study for comparison. The number of data points within the distribution has decreased to about 24,000 , but the standard deviation of the distribution has again significantly decreased and the number of data points within $45^{\circ}$ of anti-parallel alignment is now well over half the total number of data points. The 


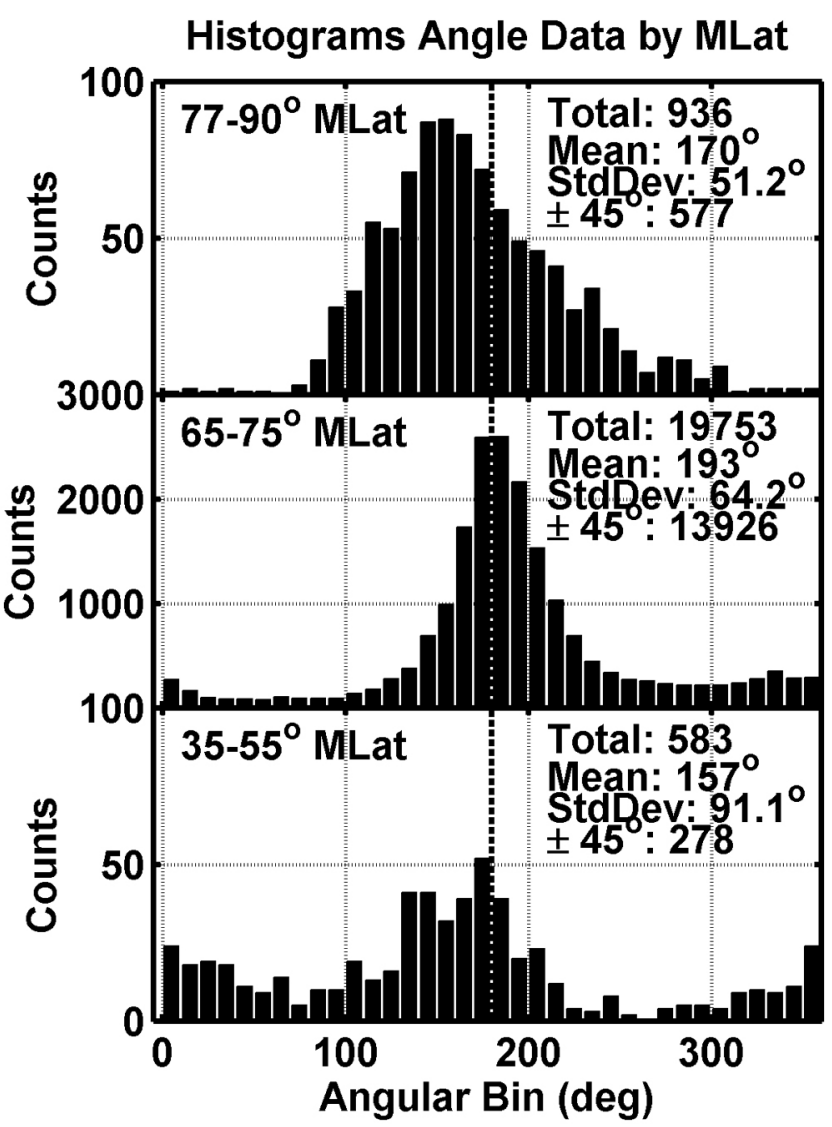

Figure 3. Histograms of angle between equivalent ionospheric currents and SuperDARN flow vectors binned by magnetic latitude. (top) The distribution for equivalent ionospheric currents and SuperDARN flow vectors obtained between $77^{\circ}$ and $90^{\circ}$ MLat. (middle) The distribution obtained between $65^{\circ}$ and $75^{\circ}$ MLat. (bottom) The distribution determined between $35^{\circ}$ and $55^{\circ}$ MLat. The top and bottom panels have different $y$-scales than the middle panel. Those two distributions are nearly empty because few large equivalent ionospheric currents and SuperDARN flows are measured at those magnetic latitudes.

peak to background ratio has also increased to about 13 , but the mean angle between the currents and flow is now $191^{\circ}$.

\section{Discussion}

[16] The results of section 3 indicate that the best antiparallel alignment occurs when small values of the equivalent ionospheric currents and flow magnitudes are eliminated and when we examine only measurements nearly collocated in space. The distribution in Figure 2 (middle) also suggests that the stronger the equivalent ionospheric currents and flow vectors the better their direction is determined. We find that increasing the cutoff values for distributions similar to Figure 2 (middle) does reduce the standard deviation, but does not consistently give a mean alignment of $180^{\circ}$. For the remainder of the study we will examine the effects of magnetic latitude, geomagnetic activity, magnetic local time, and season.
[17] Figure 3 displays the distributions of the angles between the vectors as a function of the magnetic latitude. In all three panels we have again limited the observations to currents larger than $64.2 \mathrm{~mA} / \mathrm{m}$, flows larger than $100 \mathrm{~m} / \mathrm{s}$, and observations close to magnetometer stations as defined in section 3. Figure 3 (top) consists of observations above $77^{\circ}$ magnetic latitude in what is typically the polar cap region. Figure 3 (middle) has angles obtained between $65^{\circ}$ and $75^{\circ}$ magnetic latitude in a region that normally contains the auroral oval and Figure 3 (bottom) shows the distribution for angles obtained between $35^{\circ}$ and $55^{\circ}$ magnetic latitude, which would be normally well outside the auroral oval. The bulk of the data points occur within the auroral oval region where the currents are strongest, the bulk of the magnetometers are distributed, and the SuperDARN radars typically have the most backscatter.

[18] It is interesting to note that none of the means in these three panels are really anti-parallel and that only at the auroral oval latitudes does the mean exceed $180^{\circ}$. We speculate that none of the distribution peak at $180^{\circ}$ because of Pedersen currents contributing to the equivalent ionospheric currents.

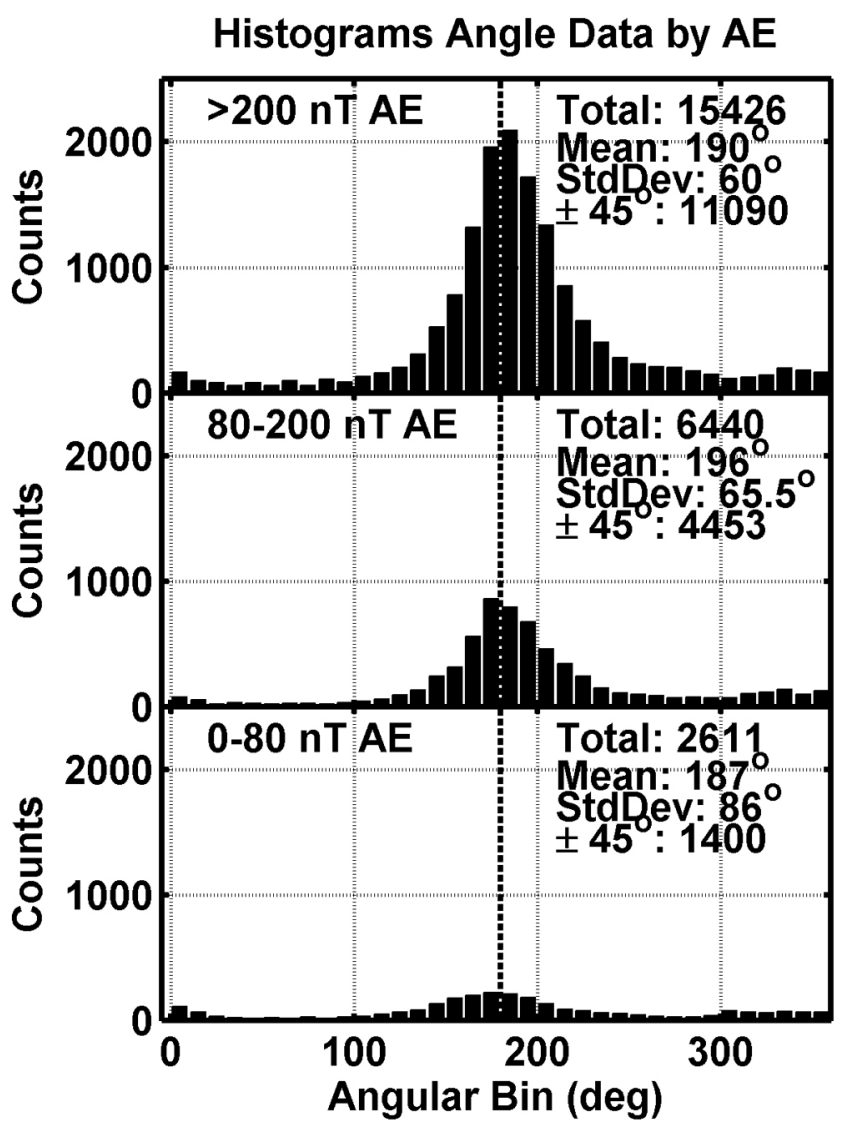

Figure 4. Histograms of angle between equivalent ionospheric currents and SuperDARN flow vectors binned by geomagnetic activity. (top) The distribution for equivalent ionospheric currents and SuperDARN flow vectors obtained for active geomagnetic conditions (AE > $200 \mathrm{nT}$ ). (middle) The distribution was determined for moderate geomagnetic activity (AE between 80 and $200 \mathrm{nT}$ ). (bottom) The distribution derived during quiet conditions (AE between 0 and $80 \mathrm{nT})$. 

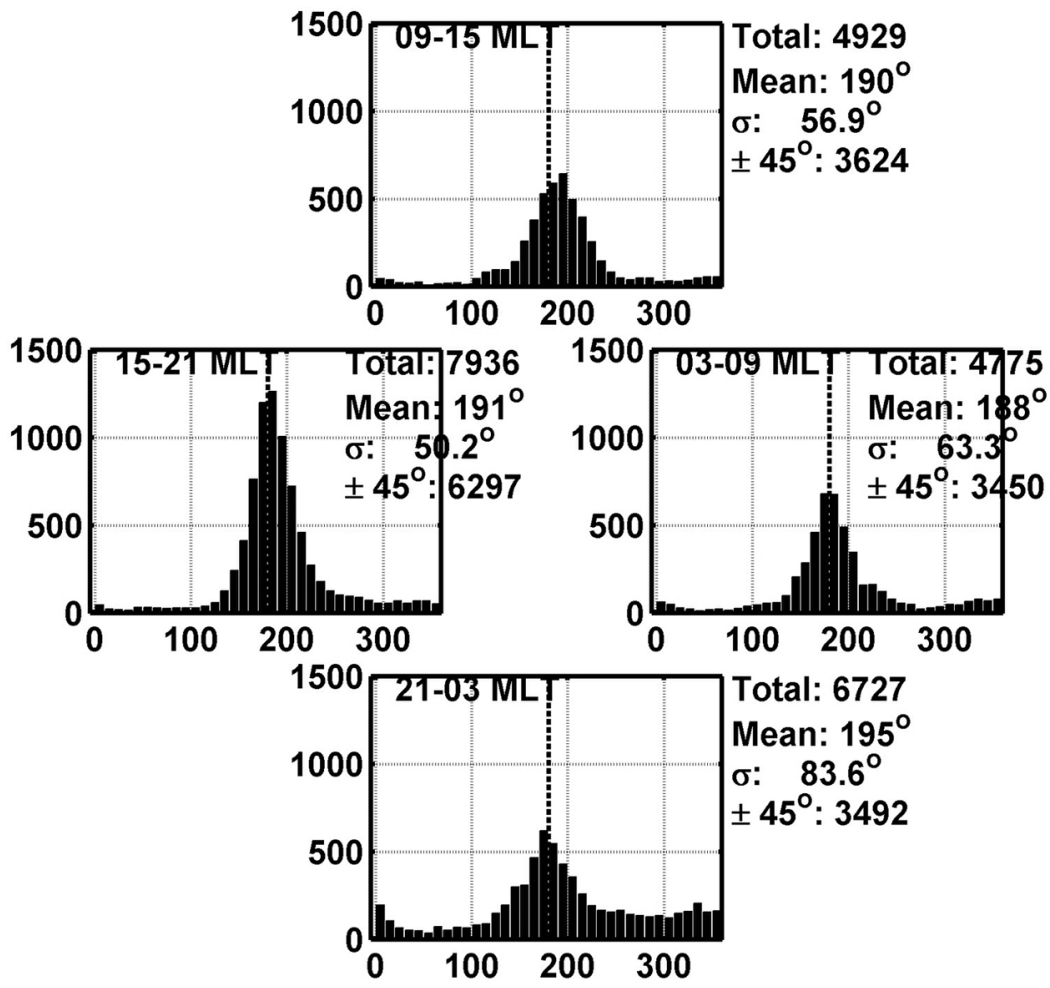

Figure 5. Histograms of angle between equivalent ionospheric currents and SuperDARN flow vectors sorted by magnetic local time. (top and bottom) The distribution associated with the noon (09 to $15 \mathrm{MLT}$ ) and midnight sectors (21 to $03 \mathrm{MLT}$ ), respectively. (left and right) The distributions for the dusk (15 to 21 MLT) and dawn sectors (03 to 09 MLT), respectively.

A much larger data set of near simultaneous equivalent ionospheric currents and ionospheric flows should be developed to more rigorously investigate this idea. The data set should be large enough to be able to bin the data simultaneously by MLat, MLT, and AE to determine if a systematic influence of the Pedersen currents is present in the distributions. In addition to the absence of peak at $180^{\circ}$ for each distribution, the standard deviation for Figure 3 (top) is significantly smaller than Figure 3 (middle) and has considerably fewer data points for angles between $0^{\circ}$ and $100^{\circ}$ as well as between $260^{\circ}$ and $360^{\circ}$. The standard deviation may be the largest for the subauroral region and smallest in the top and middle panels due the area covered by radars. The fields of view of most of the SuperDARN radars overlap in the auroral oval and polar cap region, but few observe backscatter from the lower latitudes.

[19] Figure 4 shows the distribution of the angles as a function of geomagnetic activity. Figure 4 (top) shows the results for active AE greater than $200 \mathrm{nT}$, Figure 4 (middle) shows the results for moderate AE between $80 \mathrm{nT}$ and $200 \mathrm{nT}$, and Figure 4 (bottom) shows the results for quiet $\mathrm{AE}$ less than $80 \mathrm{nT}$. These $\mathrm{AE}$ ranges were arbitrarily selected such that there are approximately an equal number of AE values per bin. Figure 4 (top) with the largest geomagnetic activity and, by definition, the strongest electrojet currents has the smallest standard deviation and a mean alignment of $190^{\circ}$. In Figure 4 (middle and bottom), where the geomagnetic activity is weaker, the standard deviation significantly increases and the alignment in the middle panel gets worse $\left(196^{\circ}\right)$. However, the alignment between the flows and currents in the bottom panel is $187^{\circ}$ and closer to $180^{\circ}$ than the other distribution. In general, this supports our earlier assertion that the stronger the equivalent ionospheric currents and flow vectors are the better their direction is determined.

[20] In Figure 5 we display the distribution of the angles as a function of the magnetic local time (MLT). The figure helps us investigate the statement that the equivalent ionospheric currents do not have the spatial resolution in regions where the ionospheric currents and flow patterns are complex as auroral images and ionospheric radar measurements would suggest. We would expect the smallest standard deviations to be in the dawn (03 to 09 MLT), dusk (15 to 21 MLT), and noon (09 to 15 MLT) sectors where the ionospheric currents and convection patterns are relatively simple and largest standard deviations in the midnight sector where the ionospheric current patterns are more complex and that is what we find. In the dawn, dusk, and noon sectors of Figure 5 the standard deviation and alignment is better than Figure 2 (bottom) and the standard deviation and alignments is considerably worse in the midnight sector. This finding does not necessarily mean that the methods for determining the currents and flow are poor, but that the flows and currents have more spatial detail than is being captured by the techniques.

[21] Figure 5 of this study also is a near-reproduction of the procedure used to produce Figure 5 of Benkevitch et al. [2006]. Our results are in some respects better and in some respects not as good as the Benkevitch ones. In terms of the standard deviation of the distribution our study has 


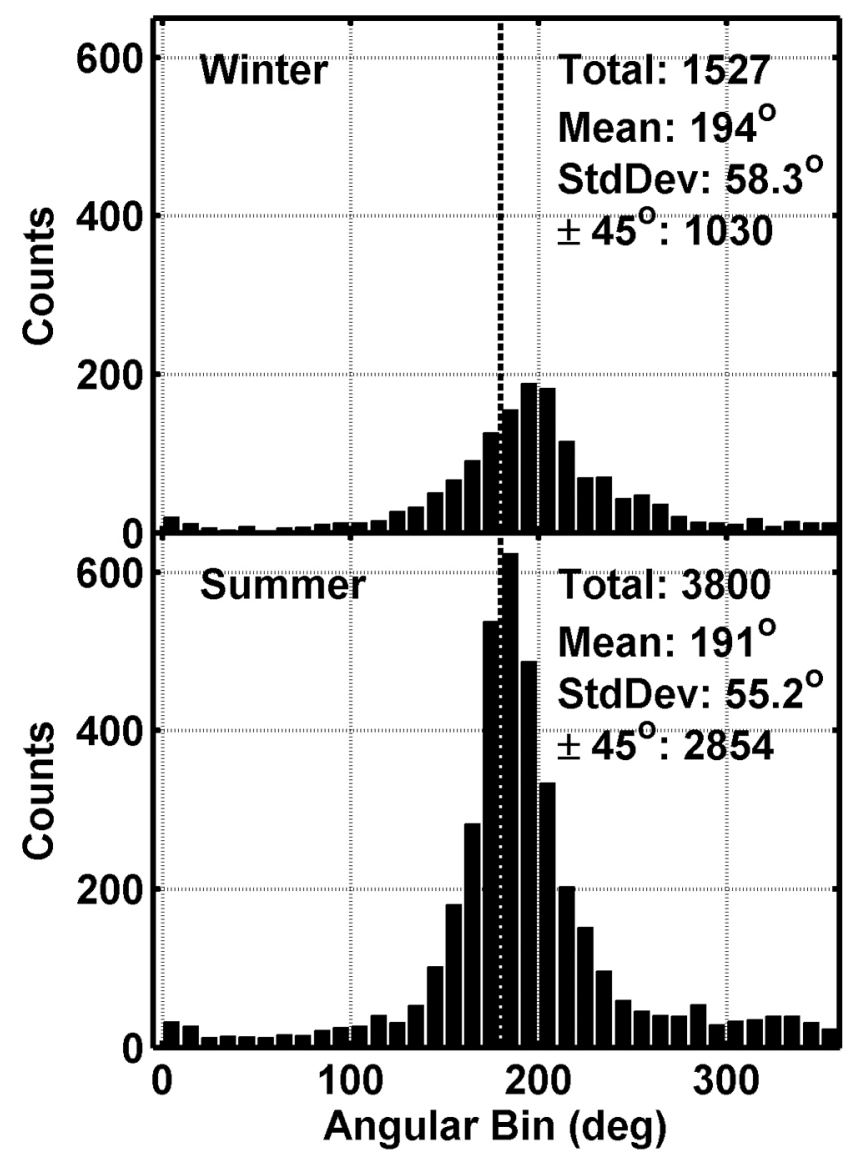

Figure 6. Histograms of angle between equivalent ionospheric currents and SuperDARN flow vectors binned by season in the northern hemisphere. (top) Distribution of the angles during the winter season. (bottom) Distribution for the summer season.

significantly smaller standard deviations in all sectors except the midnight sector. Even the order of the standard deviations for each sector from smallest to largest was the same in our study as in the Benkevitch et al. [2006] study. However, the difference between the anti-parallel alignments in our study was not as good as in Benkevitch et al. [2006]. In our study the mean difference between the flow vector and the current vector anti-parallel alignment was about $10^{\circ}$ and always greater than $180^{\circ}$ of anti-parallel, but in the Benkevitch et al. [2006] study the difference between the flow vector and the magnetic equivalent convection was about $5.5^{\circ}$ on either side of parallel alignment. The reason for this misalignment in our work is unclear at this time.

[22] In Figure 6 we examine the effects of ionospheric conductivity on the anti-parallel agreement between the equivalent ionospheric currents and the ionospheric flow vectors. The figure shows the distribution of the angle between the two vectors for both the winter and the summer seasons in Figure 6 (top) and Figure 6 (bottom), respectively. We assume higher conductivities exist in summer due to the higher degree of ionospheric ionization. In addition to restricting the derived current to near magnetometer stations and eliminating small current and flow magnitudes, we have also eliminated measurements below $65^{\circ}$ geographic latitude.
This weak restriction still does include some sunlit and dark observation in each distribution; however setting the cutoff latitude to high values leaves too few data points in the distributions. A much larger study is needed if we are to restrict ourselves to only a sunlit or dark ionosphere.

[23] These seasonal distributions are similar to the result of Benkevitch et al. [2006]. They found the best agreement between the magnetic equivalent convection and flow occurred in the summer when the hemisphere was sunlit, the conductivity is higher, and the conductivity gradients smaller than in the winter. The standard deviation derived in Benkevitch et al. [2006] was also significantly lower and the peak to background ratio was about 20 in the summer and about 10 in the winter. Our results agree, but not as strongly. The best alignment occurs in the summer with an alignment of $191 \pm 1^{\circ}$. This is not that different from our winter alignment of $194 \pm 1^{\circ}$, but still significantly different by only a few degrees. However, the peak to back ground ratio for our summer distribution, which is 22 , is much better than in the winter histogram where it is 16 . The differences between our study and the Benkevitch et al. [2006] study may be due to a couple of reasons. The first reason may be related to our inclusion of sunlit ionosphere in the winter distribution and vice versa for the summer distribution. However, the Benkevitch et al. [2006] study also had some mixing of the two as well so this point may not be as critical as we believe. Second, the solar cycle may play some role. The results from the Benkevitch et al. [2006] study were obtained during the peak of the solar cycle while our results were obtained from nearly the minimum of solar cycle. It is has been shown that the ionospheric conductivity varies with solar cycle [Volland, 1995] and this may have influenced our results.

[24] Despite binning our angles between the currents and the flows by season, magnitude, magnetic latitude, and geomagnetic activity there is always a significant spread in the distribution and an ever present noise threshold. This spread of the distribution and noise threshold may be the result of non-uniform ionospheric conductivity (most important), poorly defined quiet time backgrounds for each magnetometer, and the method of determining the flow vectors. Radar observations and auroral images suggest that the ionospheric conductance values can vary significantly over scales of just a few tens of kilometers [Tsunoda, 1988; Paschmann et al., 2003]. This means that the Fukushima theory for a uniform conducting ionosphere, which states that a ground magnetometer will not be able to measure the Pedersen currents [Fukushima, 1969, 1976], will not hold true and the ground magnetometer can measure a fraction of the Pedersen and the FACs. In fact, Amm [1997] demonstrated that Pedersen currents do play a small role, although small, in the determination of the equivalent ionospheric currents. Thus our assumption that the equivalent ionospheric currents are mostly Hall currents is not ideal. While this assumption for the conductance and currents appears to be detrimental to our study, the fact that the distributions of the angles are well defined with a large peak to background ratio value indicates that the SECS method can still provide a macroscopic picture of the ionospheric currents in many cases.

[25] In addition to ionospheric conductance gradients the determination of the quiet time backgrounds of the stations may play a role in the accurate determination of the currents. 
The method to determine the quiet time backgrounds is discussed in detail in Weygand et al. [2011]; however, we will briefly summarize the technique here. To obtain the quiet time backgrounds we average together all the quietest intervals (i.e., intervals with small standard deviations in the magnetic field) varying in length from $1 \mathrm{~h}$ to $24 \mathrm{~h}$. This method produces smooth backgrounds at low latitude $\left(<\sim 55^{\circ}\right)$; however, at higher latitudes, where geomagnetic activity is frequently present, the backgrounds are not as smooth and may have fluctuations on the order of $5 \mathrm{nT}$. While our quiet time backgrounds are normally smoother than the average of the 5 quietest days used by the World Data Center the small fluctuations can still influence our equivalent ionospheric currents especially during quiet geomagnetic activity. The increase of the standard deviation with decreasing geomagnetic activity shown in Figure 4 supports this hypothesis. However, the fact that we remove the weak equivalent ionospheric currents and, therefore, the effects of the magnetic perturbations in the quiet time background refutes that hypothesis. A more detailed study of the quality of the quiet time backgrounds should be done in the future.

\section{Summary and Conclusion}

[26] We have shown with the equivalent ionospheric currents derived using the SECS method and the SuperDARN ionospheric flow that over $66 \%$ of the time the ionospheric current vectors are within $45^{\circ}$ of anti-parallel of the flow and in many of the subsets of the data over $70 \%$ of the time the ionospheric current vectors are within $45^{\circ}$ of anti-parallel. The best anti-parallel alignments occurred at auroral latitudes, during high levels of geomagnetic activity when the currents are well determined in the dawn, dusk, and noon sectors in the northern hemisphere summer season. We believe the best agreement occurs under these conditions because the ionospheric currents are largest and well determined by the ground magnetometers in auroral regions where the ionospheric currents and flow patterns are simple and do not contain a significant amount of small-scale structures. The most significant sources of error for the current vectors are most likely ionospheric conductance gradients, merging of the flow vectors, errors in the radar measurements, and the determination of the quiet time background for the magnetometers. This study used only three months of equivalent ionospheric currents and flow vectors; however, a much larger study could be done to more thoroughly investigate the role of parameters examined within this study.

[27] In this study we have shown that equivalent ionospheric currents could be used to obtain a macroscopic picture of the ionospheric convection over North America when ionospheric flows are not available. However, ionospheric currents combined with the THEMIS all sky mosaics, coherent and incoherent scatter radar data, and spacecraft data could be used to investigate magnetosphere-ionosphere coupling with more detail and clarity than in the past.

[28] Acknowledgments. We thank the many different groups operating magnetometer arrays for providing data for this study including: the Canadian Space Science Data Portal. The Canadian Space Science Data Portal is funded in part by the Canadian Space Agency, the Alberta Science and Research Authority, and the University of Alberta. The Canadian Magnetic Observatory Network (CANMON) is maintained and operated by the Geological Survey of Canada: http://gsc. nrcan.gc.ca/geomag. The Magnetometer Array for Cusp and Cleft Studies (MACCS) array is supported by U.S. National Science Foundation grant ATM-0827903 to Augsburg College. We would like to would like to thank the following: M. J. Engebretson, D. Murr, and E. S. Steinmetz at Augsburg College and the MACCS team. The Solar and Terrestrial Physics (STEP) magnetometer file storage is at Department of Earth and Planetary Physics, University of Tokyo and maintained by Kanji Hayashi (hayashi@grl.s.u-tokyo.ac.jp). This study was made possible by NASA THEMIS grant SA3650 at UCLA and grant NE/G019665/1 for supporting Adrian Grocott. We would also like to thank M. G. Kivelson, K. K. Khurana, R. J. Walker, A. Pulkkinen, and L. V. Benkevitch for their invaluable input. [29] Robert Lysak thanks the reviewers for their assistance in evaluating the paper.

\section{References}

Amm, O. (1997), Ionospheric elementary current systems in spherical coordinates and their application, J. Geomagn. Geoelectr., 49, 947-955, doi:10.5636/jgg.49.947.

Amm, O., and A. Viljanen (1999), Ionospheric disturbance magnetic field continuation from the ground to the ionosphere using spherical elementary currents systems, Earth Planets Space, 51, 431-440.

Benkevitch, L. V., A. V. Koustov, J. Liang, and J. F. Waterman (2006), Comparison of the magnetic equivalent convection direction and ionospheric convection observed by the SuperDARN radars, Ann. Geophys., 24, 2981-2990, doi:10.5194/angeo-24-2981-2006.

Chapman, S., and J. Bartels (1940), Geomagnetism, vol. 2, Oxford Univ. Press, London.

Chisham, G., et al. (2007), A decade of the Super Dual Auroral Radar Network (SuperDARN): Scientific achievements, new techniques and future directions, Surv. Geophys., 28, 33-109, doi:10.1007/s10712-007-9017-8.

Engebretson, M. J., W. J. Hughes, J. L. Alford, E. Zesta, L. J. Cahill Jr., R. L. Arnoldy, and G. D. Reeves (1995), MACCS observations of the spatial extent of broadband ULF magnetic pulsations at cusp/cleft latitudes, J. Geophys. Res., 100, 19,371-19,386, doi:10.1029/95JA00768.

Fukushima, N. (1969), Equivalence in ground geomagnetic effect of Chapman-Vestine's and Birkeland-Alfven's current systems for polar magnetic storms, Rep. Ionosph. Space Res. Jpn., 23, 219-227.

Fukushima, N. (1976), Generalized theorem for no ground magnetic effect of vertical currents connected with Pedersen currents in the uniformconductivity ionosphere, Rep. Ionosph. Space Res. Jpn., 30, 35-50.

Greenwald, R. A., et al. (1995), DARN/SuperDARN: A global view of the dynamics of high-latitude convection, Space Sci. Rev., 71, 761-796, doi:10.1007/BF00751350.

Kamide, K. (1988), Electrodynamics Processes in the Earth's Ionosphere and Magnetosphere, Kyoto Sangyo Press, Kyoto, Japan.

Kosch, M. J., O. Amm, and M. W. Scourfield (2000), A plasma vortex revisited: The importance of including ionospheric conductivity measurements, J. Geophys. Res., 105, 24,889-24,898, doi:10.1029/2000JA900102.

Lester, M., A. J. Coates, R. A. Harrison, D. Rees, J. G. Roederer, M. J. Rycroft, and M. A. Saunders (1993), International solar terrestrial energy programme and the UK participation, Surv. Geophys., 14, 555-583, doi:10.1007/BF00666030.

Lummerzheim, D., M. H. Rees, J. D. Craven, and L. A. Frank (1991), Ionospheric conductances derived from DE-1 auroral images, J. Atmos. Terr. Phys., 53, 281-289, 291-292, doi:10.1016/0021-9169(91)90112-K.

Lyatsky, W., A. V. Kustov, G. J. Sofko, B. Jacobsen, D. Andre, and L. L. Cogger (1999), Ionospheric convection and equivalent ionospheric currents in the dayside high-latitude winter ionosphere, J. Geophys. Res., $104,22,525-22,534$.

Mann, I. R., et al. (2008), The upgrade CARISMA magnetometer array in the THEMIS era, Space Sci. Rev., 141, 413-451, doi:10.1007/s11214008-9457-6.

Paschmann, G., S. Haaland, and R. Treumann (2003), Auroral Plasma Physics, ISSI Space Sci. Ser., vol. 15, Kluwer Acad., Norwell, Mass.

Press, W. H., B. P. Flannery, S. A. Teukolsky, and W. T. Vetterling (1992), Singular value decomposition, in Numerical Recipes in FORTRAN: The Art of Scientific Computing, 2nd ed. sec. 2.6, pp. 51-63, Cambridge Univ. Press, Cambridge, U. K.

Richmond, A. D., et al. (1988), Mapping electrodynamic features of the high-latitude ionosphere from localized observations: Combined incoherent-scatter radar and magnetometer measurements for January 18-19, 1984, J. Geophys. Res., 93(A6), 5760-5776, doi:10.1029/ JA093iA06p05760.

Ruohoniemi, J. M., and K. B. Baker (1998), Large-scale imaging of highlatitude convection with Super Dual Auroral Radar Network HF radar observations, J. Geophys. Res., 103, 20,797-20,811, doi:10.1029/ 98JA01288.

Russell, C. T., P. J. Chi, D. J. Dearborn, Y. S. Ge, B. Kuo-Tiong, J. D. Means, D. R. Pierce, K. M. Rowe, and R. C. Snare (2008), THEMIS ground-based 
magnetometers, Space Sci. Rev., 141, 389-412, doi:10.1007/s11214-0089337-0.

Tsunoda, R. T. (1988), High-latitude F region irregularities: A review and synthesis, Rev. Geophys., 26, 719-760, doi:10.1029/RG026i004p00719.

Untiedt, J., R. Pellinen, F. Küppers, H. J. Opgenoorth, W. D. Pelster, W. Baumjohann, H. Ranta, J. Kangas, P. Czechowsky, and W. J. Heikkila (1978), Observations of the initial development of an auroral and magnetic substorm at magnetic midnight, J. Geophys., 45, 41-65.
Volland, H. (1995), Handbook of Atmospheric Electrodynamics, vol. 2, CRC Press, Boca Raton, Fla.

Weygand, J. M., O. Amm, A. Viljanen, V. Angelopoulos, D. Murr, M. J. Engebretson, H. Gleisner, and I. Mann (2011), Application and validation of the spherical elementary currents systems technique for deriving ionospheric equivalent currents with the North American and Greenland ground magnetometer arrays, J. Geophys. Res., 116 A03305, doi:10.1029/2010JA016177. 